\title{
Time to Use the Obliviate Spell for Mcisaac Score for Childhood Pharyngitis!!
}

\section{Pearl Mary Varughese ${ }^{1 *}$, Nandita $\mathbf{P a i}^{2}$ and Prakash $\mathbf{M}^{3}$}

${ }^{1}$ Pediatric Specialist, KIMS Bellerose Institute of Medical Sciences Pvt. Ltd, Kottayam, Kerala, India

${ }^{2}$ Senior Clinical Fellow in Pediatrics, Royal London Hospital, London, United Kingdom

${ }^{3}$ Assistant Professor, Department of Community Medicine, Indira Gandhi Medical

College and Research Institute, Pondicherry, India

*Corresponding Author: Pearl Mary Varughese, Pediatric Specialist, KIMS Bellerose

Institute of Medical Sciences Pvt. Ltd, Kottayam, Kerala, India.
Received: November 25, 2020

Published: December 28, 2020

(C) All rights are reserved by Pearl Mary

Varughese., et al.

\begin{abstract}
Background: Though most common aetiology of acute pharyngitis is viral, antibiotics are being started aimlessly in most cases without prior blood tests or throat swab culture reports. This not only causes unnecessary use of antibiotics, but also antibiotic resistance in the long run.

Objective: The primary objective was to validate McIsaac score for identification of bacterial pharyngitis. The secondary objective was to put forward an easy pragmatic clinical scoring system for diagnosing GAS infection in children.

Materials and Methods: This was an observational study conducted over a 24- month period. The inclusion criteria included all the children 3-15 years who visited the hospital with acute pharyngitis. Exclusion criteria included those on antibiotics or unidentified medication in the previous week, immunocompromised, or later found to have sinusitis, otitis media or pneumonia. Sensitivity, specificity and positive and negative LR was done for many contributing factors. .

Results: The throat swab culture was positive only in 59 (72.8\%) cases being Group A streptococcal pharyngitis. Palatal petechie had the best LR and a combination of factors (Positive CRP, Positive WBC; Presence of palatal petechiae and Exudates in tonsils) was a better predictive tool than McIsaac score. By using the modified score, there was only overuse of antibiotics in $5.9 \%$ cases.

Conclusion: Most of the acute pharyngitis cases are viral, requiring symptomatic management and parental reassurance. McIsaac score is not a useful tool and combination of factors (Positive CRP, Positive WBC; Presence of palatal petechiae and Exudates in tonsils) had better predictive value for diagnosing acute pharyngitis.
\end{abstract}

Keywords: McIsaac Score; Streptococcal Pharyngitis; Antibiotics

\section{Abbreviations}

GAS: Group A Streptococcal Infections; LR: Likelihood Ratio; RADT: Rapid Antigen Detection Test; IDSA: Infectious Disease Society of America.

\section{Introduction}

Acute pharyngitis is one of the leading causes of hospital visits and admissions in children. Streptococcus pyogenes (group A streptococcus, GAS), the principal bacterial pathogen of acute sore 
throat, is responsible for merely $5-30 \%$ of cases. Hence, since most cases are of viral aetiology, antibiotic coverage is not necessary [1]. Fear of the complications, pain relief or patient satisfaction often lead physicians to use antibiotic treatment for sore throat. Inappropriate use of antibiotics is a major contributor to the rising incidence of antimicrobial resistance, currently recognized as a global health problem $[2,3]$.

A definitive diagnosis of GAS pharyngitis requires identification of the bacterium in throat swab culture or rapid antigen detection test (RADT). Throat swab culture continues to be the gold standard measure, but it is costly, time consuming and not available in many peripheral hospitals. Neither conventional throat culture nor RADTs accurately differentiate acutely infected persons from asymptomatic GAS carriers with intercurrent viral pharyngitis $[4,5]$. International guidelines differ in opinions whether the decision about antibiotic therapy should be based on microbiological testing, clinical algorithm or a combination of both [6].

Clinical scoring systems have been developed to predict the likelihood of streptococcal infection among children presenting with sore throat, most popular ones being the Centor and the modified Centor scores (McIssac score) [7,8] as shown in table 1 . Nonetheless, studies evaluating the management of pharyngitis among paediatric providers have identified high rates of antibiotic prescribing [9], even for patients with negative GAS tests [10].

To the best of our knowledge, only few studies have been conducted in India to evaluate the McIsaac sore throat score. The aim of our study was to evaluate the McIsaac sore throat score, to estimate the positive rate of GAS among paediatric patients complaining of fever and sore throat and to put forward a new easy and pragmatic predictive tool for childhood pharyngitis thus avoiding overzealous use of antibiotics.

\section{Materials and Methods}

This was an observational study involving 3- to 15-year-old children who visited the hospital (both emergency and outpatient department) with acute pharyngitis over a period of 24 months. Exclusion criteria included those who had been taking antibiotics or unidentified medication in the previous week, were immuno- compromised, or later found to have sinusitis, otitis media or pneumonia and parental refusal for throat swab. This analytic study was divided into two groups based on the data collected - Group A had retrospective data of 185 children and Group B was prospective data collection. Personal details were kept confidential and parental consent was obtained. This study was approved by the hospital Ethics Committee.

In Group A, 185 children were included in the study who visited the hospital during the period of Jan 2017- Jan 2018 with complaints of sore throat and fever and for whom the throat swab cultures were taken. Retrospective data was obtained through review of chart documentation, including notes, vital signs, lab results, and prescriptions. McIssac score was given for the patients depending on the information recorded in the patient records. There was no way of knowing whether these clinicians had knowledge of McIssac score. In Group B, 365 children who visited the hospital during Feb 2018- Feb 2019 were screened and 190 children were included. The data collection has been shown in figure 1. Patients were analysed with the McIssac score as shown in table 1 and antibiotics was prescribed accordingly once the throat swab results were confirmed.

\begin{tabular}{|c|c|}
\hline Criteria & Point \\
\hline Temperature $>38^{\circ} \mathrm{C}$ & 1 \\
No cough & 1 \\
Tender anterior cervical adenopathy & 1 \\
Tonsillar swelling or exudate & 1 \\
Age 3-14y old & 1 \\
Age 15-44y old & 0 \\
\hline A point is assigned according to patients signs and & \\
symptoms. & \\
\hline
\end{tabular}

Table 1: Mcisaac score for acute pharyngitis.

(C) McIsaac WJ, White D, Tannenbaum D, Low DE. A clinical score to reduce unnecessary antibiotic use in patients with sore throat. CMAJ., 1998, 158, 75-83 [8]. 
Other criteria factors like presence of petechie, absence of coryza, positive contact history and increased CRP and total counts were also taken into account from both the groups. (Group A had some missing data as it was retrospectively collected).

\section{Throat swab culture}

A cotton-tipped throat swab was used to swab the tonsillar and posterior pharynx surfaces. The swabs were inoculated on $5 \%$ sheep blood agar. A bacitracin disk was placed on the inoculum, and the agar was stabbed in several areas. Plates were incubated at $35^{\circ} \mathrm{C}$ aerobically for 48 hours and examined for the presence of b-haemolytic streptococcus. Isolates with streptococcal colony morphology were classified as GAS.

\section{Statistical analysis}

Data were analysed using the software SPSS 10.0 for Windows. Sensitivity, specificity, positive LR and negative LR was done for each variable, odds ratio and ROC curves.

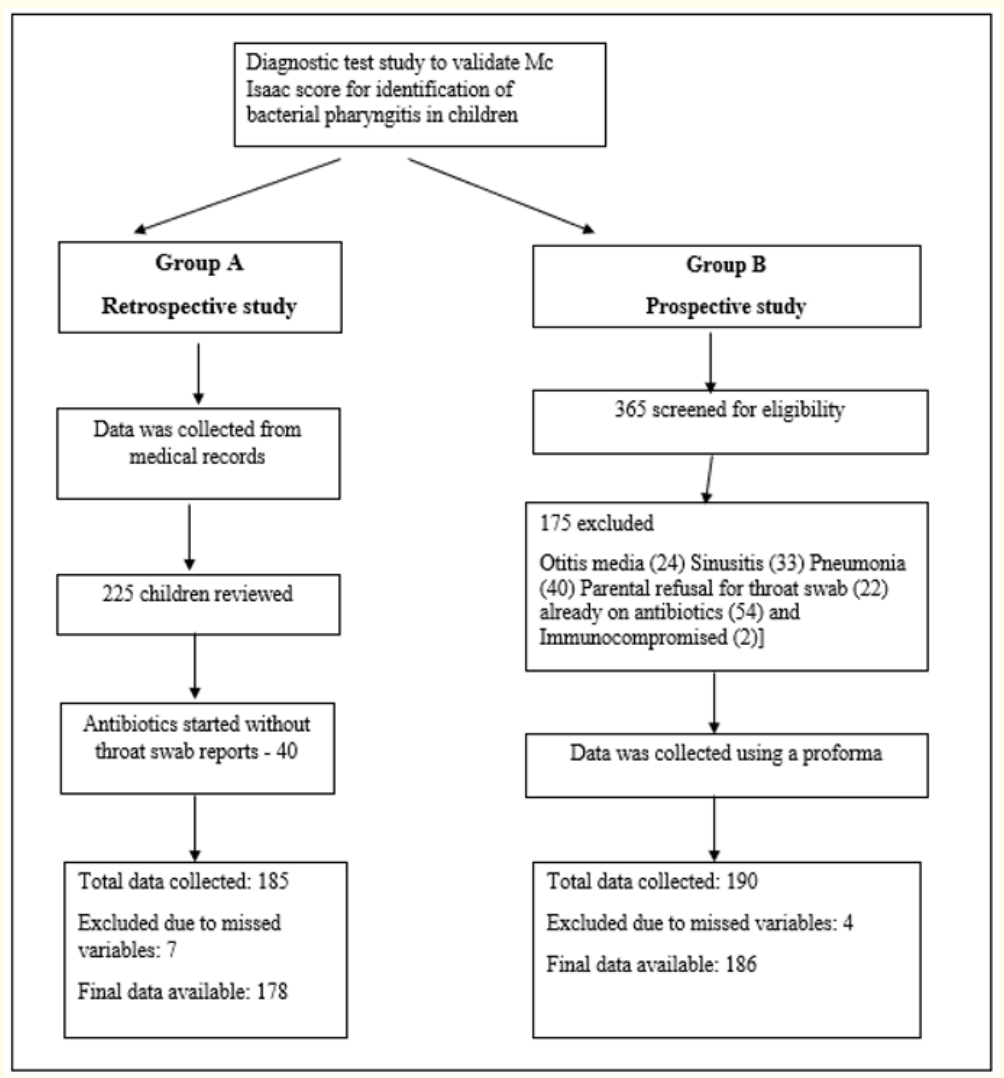

Figure 1: Data Collection.

\section{Results}

Table 2 shows the sensitivity and specificity of each of the individual factors. Factor with high sensitivity and specificity can be termed as good predicting factor. Only palatal petechia qualifies for this to be a good factor since it has the maximum sensitiv- ity and specificity with high +LR and low -LR. Tonsillar exudates, positive contact, positive CRP and WBC $>10000$ are some of the factors having high sensitivity and specificity. Tonsillar exudates, and WBC $>10000$ have +LR $(2-5)$ and - LR $(<0.5)$ can be termed as good predicting factors. 


\begin{tabular}{|c|c|c|c|c|c|c|c|}
\hline Factor & Group & Positive & Negative & Sensitivity (95\% CI) & Specificity (95\% CI) & $L R+(95 \% C I)$ & LR- $(95 \% \mathrm{CI})$ \\
\hline \multirow[t]{2}{*}{ Age } & $</=8$ & 32 & 169 & \multirow{2}{*}{$\begin{array}{c}0.5424(0.4166 \text { to } \\
0.663)\end{array}$} & \multirow{2}{*}{$\begin{array}{c}0.4652(0.4109 \text { to } \\
0.5203)\end{array}$} & \multirow{2}{*}{$\begin{array}{l}1.014(0.785 \\
\text { to } 1.31)\end{array}$} & \multirow{2}{*}{$\begin{array}{c}0.984(0.727 \text { to } \\
1.33)\end{array}$} \\
\hline & $>8$ & 27 & 147 & & & & \\
\hline \multirow[t]{2}{*}{ Sex } & $\mathrm{F}$ & 43 & 192 & \multirow{2}{*}{$\begin{array}{c}0.7288(0.604 \text { to } \\
0.8256)\end{array}$} & \multirow{2}{*}{$\begin{array}{c}0.3924(0.3402 \text { to } \\
0.4472)\end{array}$} & \multirow{2}{*}{$\begin{array}{c}1.200(1.003 \\
\text { to } 1.435)\end{array}$} & \multirow{2}{*}{$\begin{array}{c}0.69(0.445 \text { to } \\
1.073)\end{array}$} \\
\hline & M & 16 & 124 & & & & \\
\hline \multirow[t]{2}{*}{ Duration } & $>3$ days & 18 & 100 & \multirow{2}{*}{$\begin{array}{c}0.3051(0.2025 \text { to } \\
0.4315)\end{array}$} & \multirow{2}{*}{$\begin{array}{c}0.6835(0.6303 \text { to } \\
0.7324)\end{array}$} & \multirow{2}{*}{$\begin{array}{c}0.964(0.635 \\
\text { to } 1.464)\end{array}$} & \multirow{2}{*}{$\begin{array}{c}1.017(0.845 \text { to } \\
1.223)\end{array}$} \\
\hline & $</=3$ days & 41 & 216 & & & & \\
\hline \multirow[t]{2}{*}{ Duration } & $>2$ days & 50 & 230 & \multirow{2}{*}{$\begin{array}{c}0.8475(0.7348 \text { to } \\
0.9176)\end{array}$} & \multirow{2}{*}{$\begin{array}{c}0.2722(0.226 \text { to } \\
0.3237)\end{array}$} & \multirow{2}{*}{$\begin{array}{c}1.164(1.025 \\
\text { to } 1.323)\end{array}$} & \multirow{2}{*}{$\begin{array}{c}0.56(0.29 \text { to } \\
] 1.05)\end{array}$} \\
\hline & $</=2$ days & 9 & 86 & & & & \\
\hline \multirow[t]{2}{*}{ Fever } & $\mathrm{Y}$ & 59 & 236 & \multirow[t]{2}{*}{$1.0000(0.9389$ to 1$)$} & \multirow{2}{*}{$\begin{array}{c}0.2532(0.2084 \text { to } \\
0.3039\end{array}$} & \multirow{2}{*}{$\begin{array}{c}1.339(1.256 \\
\text { to } 1.428)\end{array}$} & \multirow[t]{2}{*}{0.000} \\
\hline & $\mathrm{N}$ & 0 & 80 & & & & \\
\hline \multirow[t]{2}{*}{ Cough } & $\mathrm{N}$ & 42 & 161 & \multirow{2}{*}{$\begin{array}{c}0.7119(0.5862 \text { to } \\
0.8116)\end{array}$} & \multirow{2}{*}{$\begin{array}{c}0.4905(0.4358 \text { to } \\
0.5454)\end{array}$} & \multirow{2}{*}{$\begin{array}{c}1.397(1.15 \text { to } \\
1.698)\end{array}$} & $0.587(0.387$ to \\
\hline & $\mathrm{Y}$ & 17 & 155 & & & & \\
\hline Coryza & $\mathrm{N}$ & 52 & 175 & $0.8814(0.7748$ to & $0.4462(0.3924$ to & $1.591(1.389$ & $0.266(0.131$ to \\
\hline & $\mathrm{Y}$ & 7 & 141 & & & & \\
\hline TENDER LN & $\mathrm{Y}$ & 47 & 150 & $0.7966(0.6773$ to & $0.5253(0.470$ to & $1.678(1.411$ & 0.387 (0.23 to \\
\hline & $\mathrm{N}$ & 12 & 166 & & & to 1.996 ) & $0.648)$ \\
\hline Palatal & $\mathrm{Y}$ & 49 & 13 & $0.8596(0.7468$ to & $0.9575(0.9287$ to & 20.235 & $0.147(0.077$ to \\
\hline petechiae & $\mathrm{N}$ & 8 & 293 & & & $\begin{array}{c}(11.766 \text { to } \\
34.798)\end{array}$ & \\
\hline Tonsillar & $\mathrm{Y}$ & 53 & 111 & $0.8983(0.7954$ to & 0.6487 (0.5946 to & $2.557(2.152$ & 0.157 (0.07 to \\
\hline exudate & $\mathrm{N}$ & 6 & 205 & & & to 3039$)$ & $0.336)$ \\
\hline Factor & Group & Positive & Negative & Sensitivity (95\% CI) & Specificity $(95 \% \mathrm{CI})$ & $L R+(95 \% \mathrm{CI})$ & LR- $(95 \% \mathrm{CI})$ \\
\hline Erythematous & $\mathrm{Y}$ & 34 & 133 & $0.5763(0.4493$ to & 0.4885 (0.4283 to & $1.127(0.878$ & 0.867 (0.628 to \\
\hline & $\mathrm{N}$ & 25 & 127 & & & & \\
\hline $\begin{array}{l}\text { Throat pain/ } \\
\text { swallowing diff }\end{array}$ & $\mathrm{Y}$ & 32 & 199 & $\begin{array}{c}0.5424(0.4166 \text { to } \\
0.663)\end{array}$ & $\begin{array}{c}0.3703(0.3189 \text { to } \\
0.4248)\end{array}$ & $\begin{array}{c}0.861(0.67 \text { to } \\
1.105)\end{array}$ & $\begin{array}{c}1.236(0.904 \text { to } \\
1.69)\end{array}$ \\
\hline & $\mathrm{N}$ & 27 & 117 & & & & \\
\hline positive con- & $\mathrm{Y}$ & 36 & 103 & $0.6792(0.5452$ to & $0.6688(0.614$ to & $2.051(1.608$ & $0.480(0.322$ to \\
\hline & $\mathrm{N}$ & 17 & 208 & & & & \\
\hline Positive CRP & $\mathrm{Y}$ & 50 & 62 & $\begin{array}{c}0.847(0.7348 \text { to } \\
0.9176)\end{array}$ & $\begin{array}{c}0.8038(0.7565 \text { to } \\
0.8438)\end{array}$ & $\begin{array}{c}4.319(3.37 \text { to } \\
5.535)\end{array}$ & $\begin{array}{c}0.190(0.104 \text { to } \\
0.347)\end{array}$ \\
\hline & $\mathrm{N}$ & 9 & 254 & & & & \\
\hline $\mathrm{WBC}>10,000$ & $\mathrm{Y}$ & 57 & 64 & 0.9661 (0.8846 to & $0.7975(0.7497$ to & $4.770(3.813$ & $0.043(0.011$ to \\
\hline & $\mathrm{N}$ & 2 & 252 & & & & \\
\hline
\end{tabular}




\begin{tabular}{|c|c|c|c|c|c|c|c|}
\hline McIsaac score & $>4$ & 34 & 23 & $\begin{array}{c}0.5763(0.4493 \text { to } \\
0.6939)\end{array}$ & $\begin{array}{c}0.9272(0.8932 \text { to } \\
0.951)\end{array}$ & $\begin{array}{l}7.917(5.047 \\
\text { to } 12.42)\end{array}$ & $\begin{array}{c}0.457(0.339 \text { to } \\
0.616)\end{array}$ \\
\hline \multirow[t]{2}{*}{ McIsaac score } & $>3$ & 49 & 125 & \multirow{2}{*}{$\begin{array}{c}0.8305(0.7154 \text { to } \\
0.9052)\end{array}$} & \multirow{2}{*}{$\begin{array}{c}0.6044(0.5496 \text { to } \\
0.6568)\end{array}$} & \multirow{2}{*}{$\begin{array}{c}2.100(1.75 \text { to } \\
2.51)\end{array}$} & \multirow{2}{*}{$\begin{array}{c}0.28 \text { (0.158 to } \\
0.497)\end{array}$} \\
\hline & $</=3$ & 10 & 191 & & & & \\
\hline
\end{tabular}

Table 2: Diagnostic value of all the factors.

\begin{tabular}{|c|c|c|c|}
\hline Score & Positive & Negative & LR+ (95\% CI) \\
\hline 1 & 0 & 30 & 0 \\
\hline 2 & 0 & 79 & 0 \\
\hline 3 & 10 & 82 & $0.6532(0.4831-0.8831)$ \\
\hline 4 & 15 & 102 & $0.7876(0.6558-0.9459)$ \\
\hline 5 & 34 & 23 & $7.917(6.969-8.995)$ \\
\hline
\end{tabular}

Table 3: Diagnostic value of mc issac score.

\begin{tabular}{|c|c|c|c|}
\hline Score & Positive & Negative & LR+ (95\% CI) \\
\hline 0 & 0 & 161 & 0 \\
\hline 1 & 0 & 90 & 0 \\
\hline 2 & 6 & 37 & $0.8685(0.5254-1.436)$ \\
\hline 3 & 15 & 26 & $3.09(2.477-3.854)$ \\
\hline 4 & 38 & 2 & $101.8(37.12-279)$ \\
\hline
\end{tabular}

Table 4: Diagnostic value of new modified score.

(Positive CRP, Positive WBC, Presence of palatal petechiae and Exudates in tonsils).

\begin{tabular}{|c|c|c|c|c|}
\hline \multicolumn{2}{|c|}{} & \multicolumn{2}{|c|}{ Antibiotics } & \multirow{2}{*}{ Total } \\
\cline { 3 - 5 } \multicolumn{2}{|c|}{} & $\mathbf{N}$ & $\mathbf{Y}$ & \\
\hline \multirow{2}{*}{ New modified score } & $>2$ & 4 & 77 & $81(21.6 \%)$ \\
\cline { 2 - 5 } & $</=2$ & 228 & 66 & $294(78.4 \%)$ \\
\hline \multirow{2}{*}{ McIsaac score } & $>3$ & 72 & 102 & $174(46.4 \%)$ \\
\cline { 2 - 5 } & $</=3$ & 160 & 41 & $201(53.6 \%)$ \\
\hline \multirow{2}{*}{ McIsaac score } & $>4$ & 0 & 57 & $57(15.2 \%)$ \\
\cline { 2 - 5 } & $</=4$ & 232 & 86 & $318(84.8 \%)$ \\
\hline \multirow{2}{*}{ Culture positive } & Yes & 1 & 58 & $59(15.7 \%)$ \\
\cline { 2 - 5 } & No & 231 & 85 & $316(84.3 \%)$ \\
\hline
\end{tabular}

Table 5: Antibiotics use based on the scoring system.

A LR+ bigger than 10, indicating an estimated shift in probability of at least $45 \%$, has been stated to be strongly indicative for the presence of a clinical entity, between 5 and 10 moderate (estimated shift of at least 30\%) and between 2 and 5 weak (estimated shift
15\%) [11]. Thus, while McIsaac score 5 has 7.917 as positive LR, the new modified score of 4 has 101.8 as positive LR, so it can be considered to have very high test efficiency (as shown in table 3 and 4). 


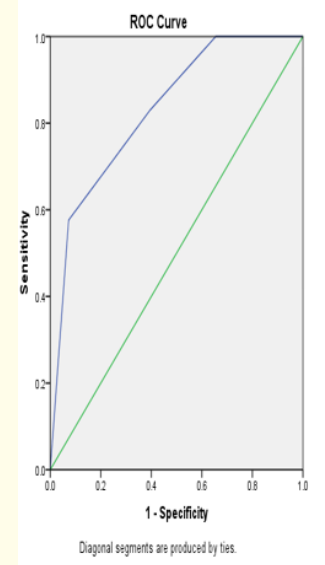

2A: McIsaac score

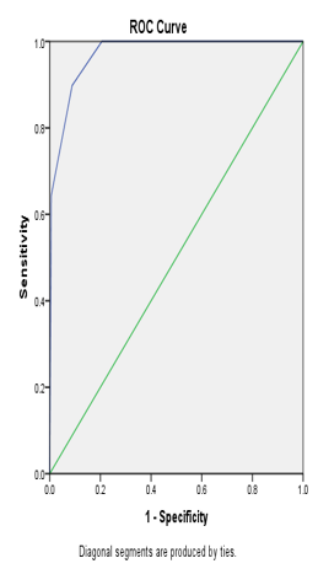

2B: New modified score

Figure 2: ROC curves.

As shown in table 5, 46.4\% of the study participants required antibiotics based on the McIsaac scoring system whereas the new modified score based on 4 points required $21.6 \%$. Actual antibiotics requirement based on culture positivity was $15.7 \%$. When the cut off of 3 was taken in McIsaac score, the antibiotics were unnecessarily used in $30.7 \%$ cases. When the cut off of 4 was taken for McIsaac score, the antibiotics use was $15.2 \%$ which in fact missed two cases of culture positive patients. That is the patients who were infected were not getting antibiotics in $0.5 \%$ cases. Meanwhile, when the new modified score was used, the difference is extra, the overuse of antibiotics is only $5.9 \%$, but there was no missing of cases. Thus, by using this modified score, we can reduce the unnecessary antibiotic prescriptions.

Area under the ROC curve is $0.830(0.777-0.884)$ and $p$ value $<0.001$ for McIsaac score while Area under the ROC curve is 0.971 (0.956 - 0.986) and $p$ value $<0.001$ for the modified new score as shown in figures $2 \mathrm{~A}$ and $2 \mathrm{~B}$ respectively.

\section{Discussion}

It is ironical that even after many years of expert strategies and policies, controversy still persists regarding the appropriate diagnosis and management of this common infection. Despite GAS being responsible for just $17 \%$ of sore throats, antibiotics are prescribed in more than $80 \%$ of cases [12]. Many clinical scoring systems are available and their predictive scores have been evalu- ated by experts worldwide, the most famous ones being the Centor score and the McIsaac score.

The original McIsaac study in Canada showed that score $\geq 4$ had $51 \%$ of predicting pharyngitis but subsequent studies in other countries showed poorer results. It also showed that antibiotic prescribing for tonsillopharyngitis is a common practice irrespective of MCC score [8,13-15]. Following this, many studies were done with the various clinical factors in different permutations and combinations to achieve a better diagnostic and predictive value. No factor was sufficiently sensitive and specific to eliminate the need for microbiologic testing in children, and subjects with all clinical features in a particular scoring system can be confirmed to have streptococcal pharyngitis only approximately $35-50 \%$ of the time. Vasudhevan., et al. concluded that the specificity was $100 \%$ for palatine petechiae, followed by palatine exudates $(97.5 \%)$ and tender anterior cervical nodes (88.6\%) to diagnose streptococcal pharyngitis [15]. When Steinhoff., et al. found statistically significant associations between positive cases and: exudate, fever above $38^{\circ} \mathrm{C}$ and palpable glands, Nandi., et al. found associations between enlarged tonsils, hyperaemia and palpable glands [16,17]. A retrospective validation study in 206,870 patients showed that presence of tonsillar exudates conferred the highest odds followed by swollen, anterior cervical lymph nodes, history of fever and absence of cough [18]. Shaikh et.al concluded that five findings increased the probability of streptococcal pharyngitis to $>50 \%$, namely scarlati- 
niform rash, palatal petechiae, pharyngeal exudate, vomiting and tender cervical nodes [3]. In our study too, individually palatal petechia had the best predictive value followed by tonsillar exudate and raised total counts, as shown in table 2 .

In a prospective study in Cape Town, combination of tonsillar swelling with either one of the factors like tonsillar exudate or absence of rhinorrhoea and cough showed $83.7 \%$ sensitivity and $32.2 \%$ specificity for GAS pharyngitis. They concluded that the Cape Town prediction or rule out missed only $15.9 \%$ GAS positive cases while the McIsaac rule missed $49 \%$ of the culture-positive children who should have been treated [19]. In our study, using the new modified score, we would not miss any GAS positive cases and were over prescribing in only $5.9 \%$ cases as shown in table 5 , our score proved to be better than Cape Town Prediction rule in not missing any cases.

Studies have also been done by combination of clinical and biochemical reports like total counts and CRP. Smeesters., et al. showed sensitivity of $71 \%$ and specificity of $82 \%$ when CRP was included, while Alper., et al. showed sensitivity of $81 \%$ and specificity of $78 \%$ when combining WBC with clinical scoring [20,21]. This was similar to our study which showed that combining WBC and CRP showed better predictive value as shown in table 4. But studies by Putto et.al and Orra., et al. showed that sensitivity and specificity decreased while WBC was added, probably stating that viral infections like adenotonsillitis can also lead to increased WBC counts $[22,23]$.

Till date, there is no universal protocol for the diagnosis and management of acute pharyngitis in children. In 2012, IDSA and American College of Physicians (ACP) suggested that RADT \pm culture when performed for every patient with a Centor score $\geq 2$ was helpful in reducing antibiotic prescribing [24-26]. A recent study performed by McIsaac., et al. concluded that antibiotic treatment based on either a RADT or culture positive for GAS could reduce unnecessary antibiotic usage for sore throats in children [27].

The present study has several limitations. Firstly, our study was performed at a single institution, which may have introduced selection bias by the attending physicians. Because we included only patients tested for GAS, we did not capture those treated empirically without testing. We excluded children under 3 because the presentation of GAS infection in this age group is variable, and appropriate management is less clearly defined. Another limitation is the inability to know for certain whether any of the clinicians for data collected in Group A were aware of the McIsaac score, which could bias the results.

\section{Conclusion}

The McIsaac sore throat score is not accurate in predicting paediatric GAS pharyngitis. We support the current strategy suggested by the American Academy of Pediatrics, and the IDSA that recommend testing (rapid test or a throat culture) in suspected cases and avoidance of testing in children with symptoms clearly consistent with a viral upper respiratory tract infection. When in doubt, a combination of factors ((Positive CRP, Positive WBC, Presence of palatal petechiae and Exudates in tonsils) along with throat culture would be the best method before planning of antibiotics in children, thus avoiding the risk of antimicrobial resistance in the long run.

\section{Acknowledgement}

I would like to thank all the children who participated in the study and their parents for giving consent to do so, without which this study would have been incomplete.

\section{Any Source of Funding}

Self- funded study.

\section{Conflict of Interest}

Nil.

\section{Author Contributions}

P.M.V contributed to the conception and design of this study; P.M.V performed experiments and collected data; P.M.V and N.P analyzed data; P.M.V. and N.P wrote the manuscript; N.P and P.M gave technical support and conceptual advice. P.M performed the statistical analysis All authors read and approved the final manuscript.

\section{Bibliography}

1. Panasiuk L., et al. "Antibiotics in the treatment of upper respiratory tract infections in Poland. Is there any improvement?". Journal of Clinical Pharmacy and Therapeutics 35.6 (2010): 665-669. 
2. Principi N., et al. "Control of antibiotic therapy in paediatric patients. II. Appropriateness of antibiotic choice in selected diseases". European Journal of Clinical Pharmacology 20.2 (1981): 119-121.

3. Shaikh N., et al. "Accuracy and precision of the signs and symptoms of streptococcal pharyngitis in children: a systematic review". The Journal of Pediatrics 160.3 (2012): 487-493.

4. Balasubramanian S., et al. "Rapid antigen diagnostic testing for the diagnosis of group A beta-haemolytic streptococci pharyngitis". National Medical Journal of India 31 (2018): 8-10.

5. Gerber MA. "Pharyngitis". Principles and Practice of Pediatric Infectious Disease (2008): 206-213.

6. Mazur E. "Management of acute streptococcal pharyngitis: still the subject of controversy". Open Medicine 8.6 (2013): 713719.

7. Centor RM., et al. "The diagnosis of strep throat in adults in the emergency room”. Medical Decision Making 1.3 (1981): 239246.

8. McIsaac WJ., et al. "A clinical score to reduce unnecessary antibiotic use in patients with sore throat". CMAJ 158.1 (1998): 75-83.

9. Linder JA., et al. "Antibiotic treatment of children with sore throat". Journal of the American Medical Association 294.18 (2005): 2315-2322.

10. Benin AL., et al. "Improving diagnostic testing and reducing overuse of antibiotics for children with pharyngitis: a useful role for the electronic medical record". The Pediatric Infectious Disease Journal 22 (2003): 1043-1047.

11. McGee S. "Simplifying likelihood ratios". Journal of General Internal Medicine 17.8 (2002): 647-650.

12. Holmberg SD and Faich GA. "Streptococcal pharyngitis and acute rheumatic fever in Rhode Island". Journal of the American Medical Association 250.17 (1983): 2307-2312.
13. Walker CL., et al. "Comparison of clinical prediction rules for management of pharyngitis in settings with limited resources". Journal of Pediatrics 149 (2006): 64e71.

14. Shih CT., et al. "Evaluation of a streptococcal pharyngitis score in southern Taiwan". Pediatrics and Neonatology 53.1 (2012): 49-54.

15. Vasudevan J., et al. "McIsaac modification of centor score in diagnosis of streptococcal pharyngitis and antibiotic sensitivity pattern of beta-hemolytic streptococci in Chennai, India". Indian Pediatrics 56.1 (2019): 49-52.

16. Steinhoff MC., et al. "Effectiveness of clinical guidelines for the presumptive treatment of streptococcal pharyngitis in Egyptian children". The Lancet 350.9082 (1997): 918-921.

17. Nandi S., et al. "Clinical score card for diagnosis of group A streptococcal sore throat". Indian Journal of Pediatrics 69 (2002): 471-475.

18. Fine AM., et al. "Large-scale validation of the Centor and McIsaac scores to predict group A streptococcal pharyngitis". Archives of Internal Medicine 172.11 (2012): 847-852.

19. Engel ME., et al. "The Cape Town clinical decision rule for streptococcal pharyngitis in children". The Pediatric Infectious Disease Journal 36.3 (2017): 250.

20. Joachim L., et al. "Pragmatic scoring system for pharyngitis in low-resource settings". Pediatrics 126.3 (2010): e608-614.

21. Alper Z., et al. "Diagnosis of acute tonsillopharyngitis in primary care: a new approach for low-resource settings". Journal of Chemotherapy 25.3 (2013): 148-155.

22. Putto A., et al. "C-reactive protein in the differentiation of adenoviral, EpsteinBarr viral and streptococcal tonsillitis in children". European Journal of Pediatrics 145.3 (1986): 204-206.

23. Orra M., et al. "The diagnostic value of "Centor criteria" in children with group a beta hemolytic streptococcus tonsillopharyngitis". European Journal of Biomedical 5.8 (2018): 43-49. 
24. Shulman ST., et al. "Clinical practice guideline for the diagnosis and management of group A streptococcal pharyngitis: 2012 update by the Infectious Diseases Society of America". Clinical Infection Disease 55.10 (2012): 1279-1282.

25. Kose E., et al. "The effect of rapid antigen detection test on antibiotic prescription decision of clinicians and reducing antibiotic costs in children with acute pharyngitis". Journal of Tropical Pediatrics 62.4 (2016): 308-315.

26. Llor C., et al. "Impact on antibiotic prescription of rapid antigen detection testing in acute pharyngitis in adults: A randomised clinical trial". British Journal of Practice 61.586 (2011): e244251.

27. McIsaac WJ., et al. "Empirical validation of guidelines for the management of pharyngitis in children and adults". Journal of the American Medical Association 291.13 (2004): 1587-1595.

\section{Assets from publication with us}

- Prompt Acknowledgement after receiving the article

- Thorough Double blinded peer review

- Rapid Publication

- Issue of Publication Certificate

- High visibility of your Published work

Website: $\underline{w w w}$.actascientific.com/

Submit Article: www.actascientific.com/submission.php

Email us: editor@actascientific.com

Contact us: +919182824667 